\title{
Lieb-Robinson bounds and out-of-time order correlators in a long-range spin chain
}

\author{
Luis Colmenarez $\odot^{*}$ and David J. Luitz ${ }^{\dagger}$ \\ Max Planck Institute for the Physics of Complex Systems, Noethnitzer Straße 38, Dresden, Germany
}

(Received 26 May 2020; accepted 16 September 2020; published 8 October 2020)

\begin{abstract}
Lieb-Robinson bounds quantify the maximal speed of information spreading in nonrelativistic quantum systems. We discuss the relation of Lieb-Robinson bounds to out-of-time order correlators, which correspond to different norms of commutators $C(r, t)=\left[A_{i}(t), B_{i+r}\right]$ of local operators. Using an exact Krylov space-time evolution technique, we calculate these two different norms of such commutators for the spin-1/2 Heisenberg chain with interactions decaying as a power law $1 / r^{\alpha}$ with distance $r$. Our numerical analysis shows that both norms (operator norm and normalized Frobenius norm) exhibit the same asymptotic behavior, namely, a linear growth in time at short times and a power-law decay in space at long distance, leading asymptotically to power-law light cones for $\alpha<1$ and to linear light cones for $\alpha>1$. The asymptotic form of the tails of $C(r, t) \propto t / r^{\alpha}$ is described by short-time perturbation theory, which is valid at short times and long distances.
\end{abstract}

DOI: 10.1103/PhysRevResearch.2.043047

\section{INTRODUCTION}

One of the most general concepts to study the dynamical properties of quantum many-body systems is the dynamics of quantum information, generalizing the spreading of all possible types of correlations in the system. Of particular interest is the dynamical spreading of local operators [1-3], which contains information about all correlation functions composed of these operators. While in relativistic systems the spreading of information is limited by the speed of light, there is no such strict limit in nonrelativistic quantum mechanics. However, it was shown by Lieb and Robinson [4] that quantum systems with short-range interactions exhibit a similar, nonuniversal speed limit, implying a causal structure. This emergent "light cone" has important consequences for the behavior of manybody interacting systems such as the area law of entanglement $[5,6]$, the decay of correlations [7,8], and the stability of topological order [9], as well as for the timescales of thermalization [10-12]. Recently, much progress has been made in establishing similar speed limits for the spreading of information in systems with long-range interactions and it is clear that also in such systems, information cannot spread infinitely fast [7,13-24]. However, it is not always clear whether the currently established analytical bounds on information spreading are tight for experimentally relevant lattice models. Power-law decaying interactions are present in several quantum simulator platforms, such as trapped ions [25,26], Rydberg atoms [27], ultracold atoms [28], and superconducting qubits [29], and it

\footnotetext{
*1colmena@pks.mpg.de

†dluitz@pks.mpg.de
}

Published by the American Physical Society under the terms of the Creative Commons Attribution 4.0 International license. Further distribution of this work must maintain attribution to the author(s) and the published article's title, journal citation, and DOI. Open access publication funded by the Max Planck Society. is therefore important to obtain tight bounds on information scrambling and thermalization timescales.

A useful measure to quantify the spreading of an initially local operator $\hat{A}_{i}(t)$ is the commutator with another local operator $\hat{B}_{i+r}$,

$$
\hat{C}(r, t)=\left\|\left[\hat{A}_{i}(t), \hat{B}_{i+r}\right]\right\|,
$$

where $\hat{B}_{i+r}$ serves as a probe and the operators $\hat{A}_{i}$ and $\hat{B}_{i+r}$ act only on sites $i$ and $i+r$ of the system, respectively. $\hat{A}_{i}(t)=$ $\exp (i \hat{H} t) \hat{A}_{i} \exp (-i \hat{H} t)$ is the operator $\hat{A}_{i}$ under time evolution in the Heisenberg picture and $\|$.$\| denotes any matrix$ norm. Vanishing $C(r, t)$ indicates that no information has traveled from site $i$ to $i+r$ at time $t$. It should be noted here that this generally depends very little on the choice of the operator in chaotic quantum many-body systems and only fine-tuned situations exist, where a dependence on the operator at short times can be observed [13].

In systems with short-range interactions, $C(r, t)$ is bounded within a "light-cone" region $t>r / v$, where $v$ is a velocity that depends on the microscopic model. This bound does not represent a strict cutoff since exponential tails exist outside the light cone [4,30,31].

Similarly, analytical bounds have been derived in systems with long-range interactions decaying as a power law $1 / r^{\alpha}$ with distance $r$. Hastings and Koma suggested a logarithmic bound $t \sim \ln (r)$ [7] for any $\alpha$.

In the case of strongly long-range systems with small $\alpha<D$ ( $D$ is the spatial dimension), the logarithmic bound is dominant [16,32]. Polynomial light cones have been proposed $[14,17,33]$ of the form $t \sim r^{(\alpha-2 D) /(\alpha-D+1)}$ in the regime $\alpha>2 D$, which consistently recovers the linear light cone in the short-range limit $\alpha \rightarrow \infty$. This bound was tightened to $t \sim r^{(\alpha-2 D) /(\alpha-D)}$ [18]. In general, large but finite $\alpha$ is consistently found to exhibit asymptotically short-range behavior $[13,19,21,23,34]$. It was argued by Gong et al. that a linear light-cone structure persists for $\alpha>D$ [15], which is also supported by numerical simulations $[13,34]$. A stochastic model of the operator spreading in long-range interacting systems 
points to linear light cones for $\alpha \geqslant D+\frac{1}{2}$ [35]. For general quantum state transfer protocols, only a weaker bound for a linear light cone for $\alpha>2 D+1$ is valid [36,37].

The analysis of analytical bounds [4,7,14$16,18,32,33,36,38]$ is mostly concerned with the operator norm $\|\hat{C}(r, t)\|_{2}$ [the largest singular value of the commutator matrix $\hat{C}(r, t)$ in Eq. (1)] because it encodes the "worst-case" scenario, namely, the fastest spreading modes in the system. On the other hand, numerical simulations of $C(r, t)$ usually employ the square of the normalized Frobenius norm, $\|\hat{C}(r, t)\|_{F}^{2}[12,13,23]$, which is the average over the square of its singular values and is directly related to the out-of-time order correlator (OTOC) [39-42] as shown in Eq. (7). In Ref. [37], a bound on the Frobenius norm was established and found to be different from the bound on the operator norm. The Frobenius norm, associated with typical states, was shown to exhibit linear light cones for $\alpha>5 / 2$, while for the operator norm, a weaker bound of $\alpha>3$ was found.

In a previous numerical study of long-range interacting spin chains [13], the asymptotic shape of the light cone of the OTOC (normalized Frobenius norm) was considered. In the present work, we are interested instead in the behavior of the operator norm of the commutator to compare the spreading of the fastest mode to that of typical modes (Frobenius norm). Interestingly, our analysis suggests that both the average and the largest singular value of $\hat{C}(r, t)$ have the same asymptotic behavior: We find a linear growth in time of $\|\hat{C}(r, t)\|_{2}$ at short times, and a power-law decay with distance at long distances with the exponent $\alpha$, which can be understood from perturbation theory.

\section{MODEL AND METHOD}

We study the isotropic one-dimensional Heisenberg $X X X$ model with long-range interactions,

$$
H=\sum_{i<j} \frac{J}{|i-j|^{\alpha}}\left(\hat{S}_{i}^{x} \hat{S}_{j}^{x}+\hat{S}_{i}^{y} \hat{S}_{j}^{y}+\hat{S}_{i}^{z} \hat{S}_{j}^{z}\right),
$$

where $S_{i}^{\gamma}=\sigma_{i}^{\gamma} / 2$ are spin- $1 / 2$ operators acting on site $i$, with $\gamma=x, y, z\left(\sigma_{i}^{\gamma}\right.$ are the corresponding Pauli matrices). The interaction exponent $\alpha$ controls the range of the interactions and we set $J=1$ throughout this paper. We do not use a rescaling of the coupling constant with system size $L$ to make the energy extensive for small $\alpha$ since this essentially only rescales our units of time. The model (2) conserves the total magnetization $S_{\text {tot }}^{z}=\sum_{i} \hat{S}_{i}^{z}$ and we focus on the largest magnetization sector $S_{\text {tot }}^{z}=0$ for even $L$ and $S_{\text {tot }}^{z}=\frac{1}{2}$ for odd $L$, to maximize the accessible system sizes. The limit $\alpha \rightarrow 0$ corresponds to allto-all interactions and $\alpha \rightarrow \infty$ is the nearest-neighbor limit, which are both integrable points of the model. There is also a special integrable point at $\alpha=2$, the so-called HaldaneShastry model $[43,44]$.

For concreteness, we consider the dynamical spreading of the local $\hat{S}_{i}^{z}(t)$ operator, probed by the commutators

$$
\hat{C}(r, t)=\left[\hat{S}_{i}^{z}(t), \hat{S}_{i+r}^{z}\right] .
$$

We note that due to the $\mathrm{SU}(2)$ symmetry of the model, all $S_{i}^{x}$, $S_{i}^{y}, S_{i}^{z}$ operators spread in the same way.

\section{A. Matrix norms of the commutator}

In order to quantify the growth of the commutator norm $C(r, t)$, we use two different matrix norms. The (normalized) Frobenius norm is defined as

$$
\|\hat{C}(r, t)\|_{F}:=\sqrt{\frac{\operatorname{Tr}\left[\hat{C}(r, t)^{\dagger} \hat{C}(r, t)\right]}{\mathcal{N}}}=\sqrt{\frac{\sum_{i} s_{i}^{2}}{\mathcal{N}}},
$$

where $s_{i}$ are the singular values of the commutator $\hat{C}(r, t)$ [and, consequently, $s_{i}^{2}$ are the eigenvalues of $\hat{C}(r, t)^{\dagger} \hat{C}(r, t)$ ].

The operator norm $\|\hat{C}(r, t)\|_{2}$, or 2-norm, is defined by the largest singular value,

$$
\|\hat{C}(r, t)\|_{2}=\sup _{\psi \in \mathcal{H}} \frac{\| \hat{C}(r, t)|\psi\rangle \|_{2}}{\||\psi\rangle \|_{2}}=\max _{i} s_{i}
$$

Therefore, the normalized Frobenius norm is always smaller than (or equal to) the operator norm,

$$
C_{2}(r, t) \geqslant C_{F}(r, t)
$$

where, for simplicity, we have denoted $C_{2}(r, t)=\|\hat{C}(r, t)\|_{2}$ and $C_{F}(r, t)=\|\hat{C}(r, t)\|_{F}$.

\section{B. Out-of-time order correlator and relation to Frobenius norm of the commutator}

Expanding the definition of the normalized Frobenius norm (4) for the commutator $\hat{C}(r, t)=\left[\hat{S}_{i}^{z}(t), \hat{S}_{i+r}^{z}\right]$ yields

$$
\begin{aligned}
C_{F}(r, t)^{2} & =\left\|\left[\hat{S}_{i}^{z}(t), \hat{S}_{i+r}^{z}\right]\right\|_{F}^{2} \\
& =\frac{1}{8}-\frac{2}{\mathcal{N}} \operatorname{Tr}\left[\hat{S}_{i}^{z}(t) \hat{S}_{i+r}^{z} \hat{S}_{i}^{z}(t) \hat{S}_{i+r}^{z}\right] .
\end{aligned}
$$

The correlation function $\frac{1}{\mathcal{N}} \operatorname{Tr}\left[\hat{S}_{i}^{z}(t) \hat{S}_{i+r}^{z} \hat{S}_{i}^{z}(t) \hat{S}_{i+r}^{z}\right]$ is known as the out-of-time order correlator (OTOC) and can be viewed as an infinite-temperature four-point function, where the partition function is given by the dimension of the Hilbert space, $Z=\mathcal{N}$.

In order to study the long-distance behavior of this quantity, it is crucial to access large enough system sizes to ensure the convergence of our results in the thermodynamic limit and we therefore use dynamical typicality [45] for computing the trace which appears in the Frobenius norm $\operatorname{Tr}\left[C(r, t)^{\dagger} C(r, t)\right]$. This method consists of replacing the trace operation by the expectation value $\left\langle\psi\left|\hat{C}(r, t)^{\dagger} \hat{C}(r, t)\right| \psi\right\rangle$, where $\psi$ is a random vector in the Hilbert space drawn from the Haar measure [46], and averaging over random vectors $|\psi\rangle$. Equation (7) is then boiled down to $\operatorname{Tr}\left[\hat{C}(r, t)^{\dagger} \hat{C}(r, t)\right]=\left\langle\psi^{\prime} \mid \psi^{\prime}\right\rangle$, where $\left|\psi^{\prime}\right\rangle=$ $C(r, t)|\psi\rangle$, up to an error that is exponentially small in the system size $L$, requiring a very small number of random vectors (typically $1, \ldots, 100)$ for large enough systems. The operation $C(r, t)|\psi\rangle$ is performed as a sequence of matrix-vector multiplications and several time propagations $e^{-i H t}|\psi\rangle$ of (intermediate) wave functions $|\psi\rangle$. These propagations can be performed efficiently using massively parallel sparse matrix 
Krylov space techniques. Technical details of this method for the calculation of the OTOC are discussed in Refs. [12,13,47].

\section{Operator norm of the commutator}

In the present paper, our main focus is on the operator norm (2-norm) of the commutator

$$
C_{2}(r, t)=\left\|\left[\hat{S}_{i}^{z}(t), \hat{S}_{i+r}^{z}\right]\right\|_{2},
$$

which corresponds to the largest eigenvalue [equivalent to the largest singular value of $C(r, t)]$ of the Hermitian form of the commutator $i C(r, t)=i\left[\hat{S}_{i}^{z}(t), \hat{S}_{i+r}^{z}\right]$. We use a matrix free implementation of the matrix vector product $|\tilde{\psi}\rangle \leftarrow i C(r, t)|\psi\rangle$, such that we never have to deal with dense matrices, and use the Lanczos algorithm to obtain the largest eigenvalue of $i C(r, t)$.

This means that we calculate

$$
\begin{aligned}
i C(r, t)|\psi\rangle & =i\left[\hat{S}_{i}^{z}(t), \hat{S}_{i+r}^{z}\right]|\psi\rangle \\
& =i \hat{S}_{i}^{z}(t) \hat{S}_{i+r}^{z}|\psi\rangle-i \hat{S}_{i+r}^{z} \hat{S}_{i}^{z}(t)|\psi\rangle \\
& =i e^{i \hat{H} t} \hat{S}_{i}^{z}\left|\psi_{2}(t)\right\rangle-i \hat{S}_{i+r}^{z} e^{i \hat{H} t} \hat{S}_{i}^{z}|\psi(t)\rangle \\
& =i e^{i \hat{H} t}\left|\psi_{3}\right\rangle-i \hat{S}_{i+r}^{z} e^{i \hat{H} t}\left|\psi_{4}\right\rangle \\
& =i\left|\psi_{3}(-t)\right\rangle-i \hat{S}_{i+r}^{z}\left|\psi_{4}(-t)\right\rangle \\
& =i\left|\psi_{3}(-t)\right\rangle-i\left|\psi_{5}\right\rangle \\
& \rightarrow|\tilde{\psi}\rangle .
\end{aligned}
$$

Here, we have used the replacements $\left|\psi_{2}\right\rangle=\hat{S}_{i+r}^{z}|\psi\rangle,\left|\psi_{3}\right\rangle=$ $\hat{S}_{i}^{z}\left|\psi_{2}(t)\right\rangle,\left|\psi_{4}\right\rangle=\hat{S}_{i}^{z}|\psi(t)\rangle$, and $\left|\psi_{5}\right\rangle=\hat{S}_{i+r}^{z}\left|\psi_{4}(-t)\right\rangle$. The matrix-free matrix-vector product involves again forward $|\psi(t)\rangle=e^{-i \hat{H} t}|\psi\rangle$ and backward $|\psi(-t)\rangle=e^{i \hat{H} t}|\psi\rangle$ realtime evolution of the wave function, very similar to the case of the OTOC [12,13], for which we employ a Krylov space technique for the matrix exponential [48-50]. Matrix-vector multiplications of $\hat{S}_{i}^{z}$ operators with wave functions are trivial since these operators are diagonal in the computational $S^{z}$ basis, and the entire algorithm thus requires only storage of a few vectors. This method gives access to the largest eigenvalue of the commutator with controlled accuracy up to system size $L=22(\mathcal{N}=705432$ in the zero-magnetization sector $)$. We note that for larger $\alpha$ and short distances $r$, the convergence of the Lanczos algorithm is particularly challenging due to small gaps in the spectrum. Lastly, for treating small systems $L<18$, the calculations were performed using full exact diagonalization.

Throughout this paper, we fix the position of the spreading operator to $i=3$ (the leftmost is indexed $i=0$ ) in such a way that distances $r=0,1, \ldots, L-4$ are accessible (using open boundaries) and the reflection of the left information front does not interfere with propagation of the right one (which is the one we study in detail).

\section{RESULTS}

In the following, we analyze in detail the space-time profile of the operator norm of the commutator $\|\hat{C}(r, t)\|_{2}$ of the long-range $X X X$ chain (2) and compare it to the case of the normalized Frobenius norm (OTOC), for which a very detailed analysis can be found in Ref. [13]. We provide additional complementary data for the $X Y Z$ chain in Appendix B.

\section{A. Causal space-time region}

We start our analysis by providing a qualitative comparison of the two norms of the commutators $\hat{C}(r, t)=\left[\hat{S}_{3}^{z}(t), \hat{S}_{3+r}^{z}\right]$ for different range of the interaction $\alpha$ and all distances $r$ as a function of time. The synopsis of these results is shown in Fig. 1, where the top row shows the operator norm and the bottom row shows the normalized Frobenius norm (OTOC), while columns correspond to different ranges of the interaction $\alpha=0.3,0.7,1.2,2.4$. Both norms are shown on the same color scale. Full lines show contour lines of the space-time profile for various thresholds $\theta$, extracted from the solution of the equation $C(r, t)=\theta$ to obtain the light "cone" $t_{\theta}(r)$. It is already clear from a visual inspection of the two norms that the essential behavior is identical. Both norms reveal a clear causal space-time region outside of which the commutator is very small, which means that almost no quantum information is communicated at short times and long distances for all $\alpha$.

The contours are calculated for the same set of threshold values $\theta$ (indicated as vertical lines in the color bar for clarity), clearly showing that the operator norm reaches a fixed threshold earlier than the Frobenius norm due to the property given by Eq. (6). Since no signal can travel faster than governed by the operator norm, it strictly limits the amount of quantum communication outside the causal region. The comparison between the operator and the Frobenius norm shows that typical modes in the system [singular values of $\hat{C}(r, t)$ ] travel significantly slower than the fastest mode (maximal singular value), an effect which is particularly pronounced at small $\alpha$, as can be seen from a comparison of the contour lines between the two norms.

The overall shape of the contour lines appears to be identical (with different prefactors). For large $\alpha$, both norms are consistent with asymptotically linear light cones.

For intermediate $\alpha=1.2$ and large thresholds (black contour lines), we observe a "bump" in the case of the operator norm, which is likely nonuniversal and stems from the reflection at the left edge of the system; therefore we focus on smaller thresholds in these cases, where reflection does not (yet) interfere due to the observed causality.

\section{B. Early time growth}

In Fig. 2, we analyze the growth of the operator norm $C_{2}(r, t)=\|\hat{C}(r, t)\|_{2}$ as a function of time $t$ for fixed distances $r$. The results are shown for a system of size $L=22$ and reveal very clearly that the operator norm grows linearly in time (linear growth shown by dashed black lines for comparison). We have checked that this short-time behavior is converged in system size. This is identical to the behavior of the normalized Frobenius norm (OTOC) [13] and is expected in the short-time perturbative regime when $t \ll 1$. We show how this linear growth arises from short-time perturbation theory in Sec. III C, where it also becomes clear that nearest-neighbor 


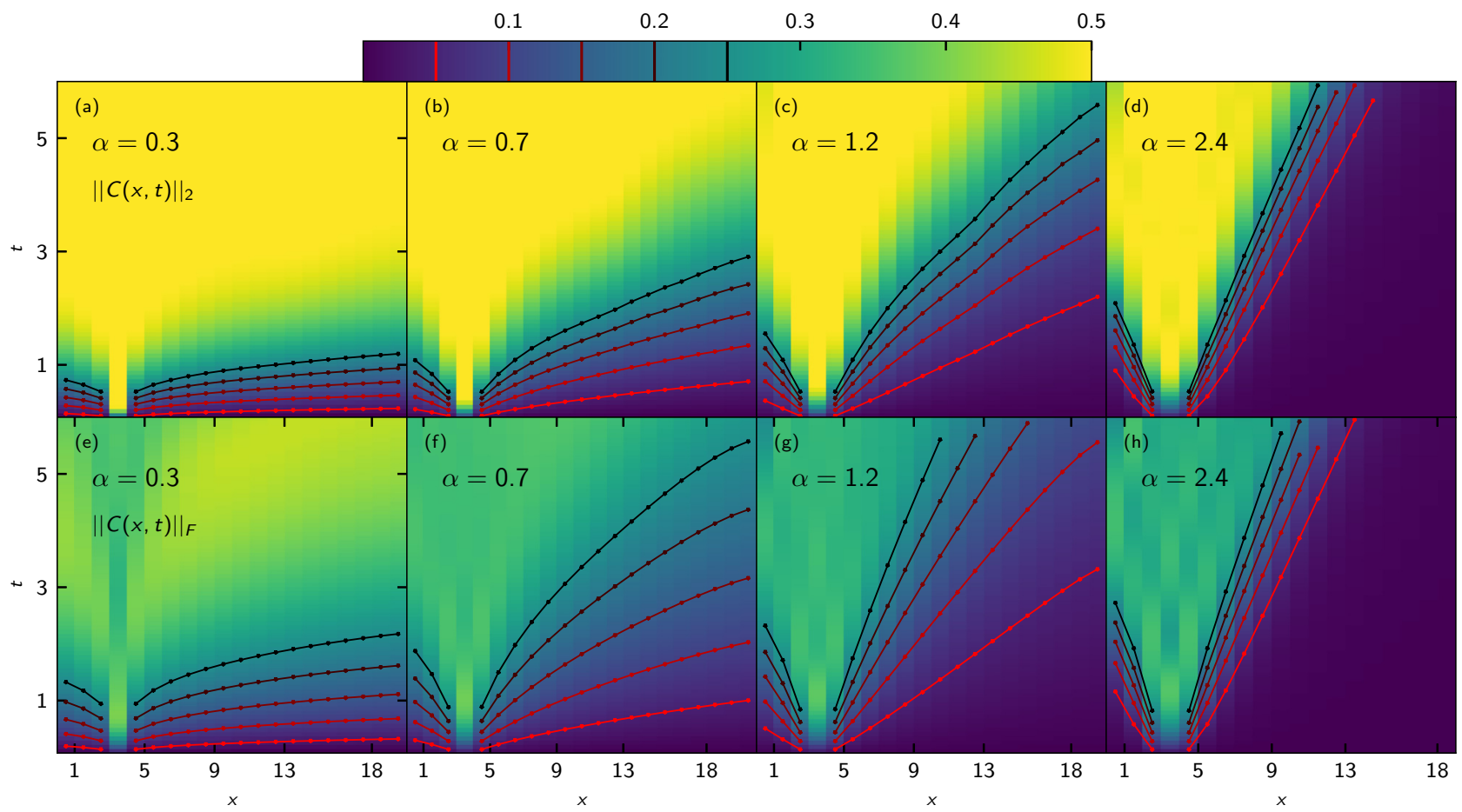

FIG. 1. Norms of the commutator $\hat{C}(x, t)=\left[\hat{S}_{i}^{z}(t), S_{x}^{z}\right]$ as a function of time $t$ and position $x$ in the chain for different interaction exponents $\alpha$. The first operator is located at $i=3$. The top row corresponds to the operator norm [largest singular value of $C(x, t)]$ and the bottom row shows the normalized Frobenius norm [root of the mean of squared singular values of $C(x, t)$ ]. The curves correspond to contour lines for different thresholds $\theta$, i.e., the solution $t_{\theta}(x)$ of the equation $\|C(x, t)\|=\theta$ with $\theta=0.05,0.1,0.15,0.2,0.25$ for both norms (lines marked in the color bar in increasing order). The interaction exponents $\alpha=0.3,0.7,1.2,2.4$ correspond to system sizes $L=21,22,21,20$, respectively.

interactions lead to a different (power-law) short-time behavior.

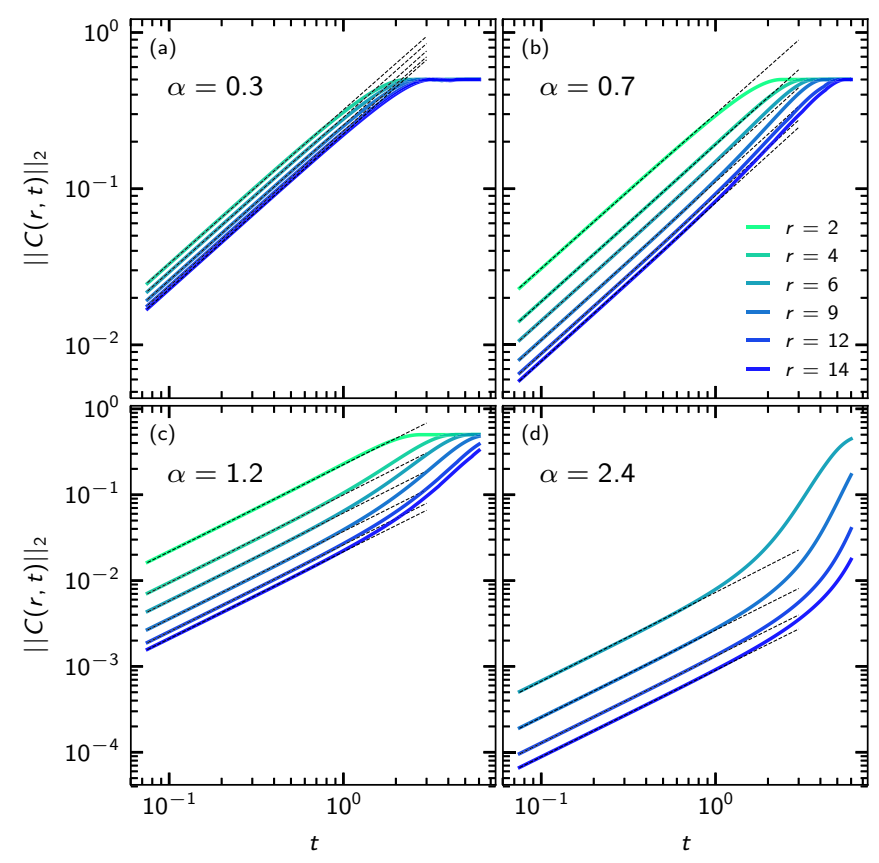

FIG. 2. $C_{2}(r, t)$ as function time at fixed distance $r$ from site $i=$ 3. Dashed lines are linear power-law fits to times $t<1$. The fitted lines are extended up to $t=3$. The system size is $L=22$.

\section{Perturbation theory in the short-time limit}

At short times, we can use the Baker-Campbell-Hausdorff (BCH) formula,

$$
e^{\hat{X}} \hat{Y} e^{-\hat{X}}=\sum_{m=0}^{\infty} \frac{1}{m !}[\hat{X}, \hat{Y}]_{m},
$$

with $[\hat{X}, \hat{Y}]_{m}=\left[\hat{X},[\hat{X}, \hat{Y}]_{m-1}\right]$ and $[\hat{X}, \hat{Y}]_{0}=\hat{Y}$. Replacing $\hat{Y}=\hat{S}_{i}^{z}$ and $\hat{X}=i t \hat{H}$, we get a perturbative expansion for time-dependent Heisenberg operators,

$$
\hat{S}_{i}^{z}(t)=\sum_{m=0}^{\infty} \frac{(i t)^{m}}{m !}\left[\hat{H}, \hat{S}_{i}^{z}\right]_{m} .
$$

The commutator given by Eq. (3) can then be written as

$$
\left[\hat{S}_{i}^{z}(t), \hat{S}_{i+r}^{z}\right]=\sum_{m=0}^{\infty} \frac{(i t)^{m}}{m !}\left[\left[\hat{H}, \hat{S}_{i}^{z}\right]_{m}, \hat{S}_{i+r}^{z}\right] .
$$

For systems with long-range interactions, the commutator to linear order, $\left[\left[\hat{H}, \hat{S}_{i}^{z}\right], \hat{S}_{i+r}^{z}\right]$, is nonzero for any distance $r$, and is therefore the leading order at short times, leading to a dominant term linear in $t$. For the long-range $X X X$ model given by Eq. (2), we obtain, for $r>0$,

$$
\left[\hat{S}_{i}^{z}(t), \hat{S}_{i+r}^{z}\right]=\frac{i t}{r^{\alpha}}\left(\hat{S}_{i}^{x} \hat{S}_{i+r}^{x}+\hat{S}_{i}^{y} \hat{S}_{i+r}^{y}\right)+\mathcal{O}\left(t^{2}\right) .
$$

Therefore, the operator norm $C_{2}(r, t)$ to leading order in $t$ reads

$$
\left\|\left[\hat{S}_{i}^{z}(t), \hat{S}_{i+r}^{z}\right]\right\|_{2}=\frac{t}{2 r^{\alpha}}+\mathcal{O}\left(t^{2}\right)
$$




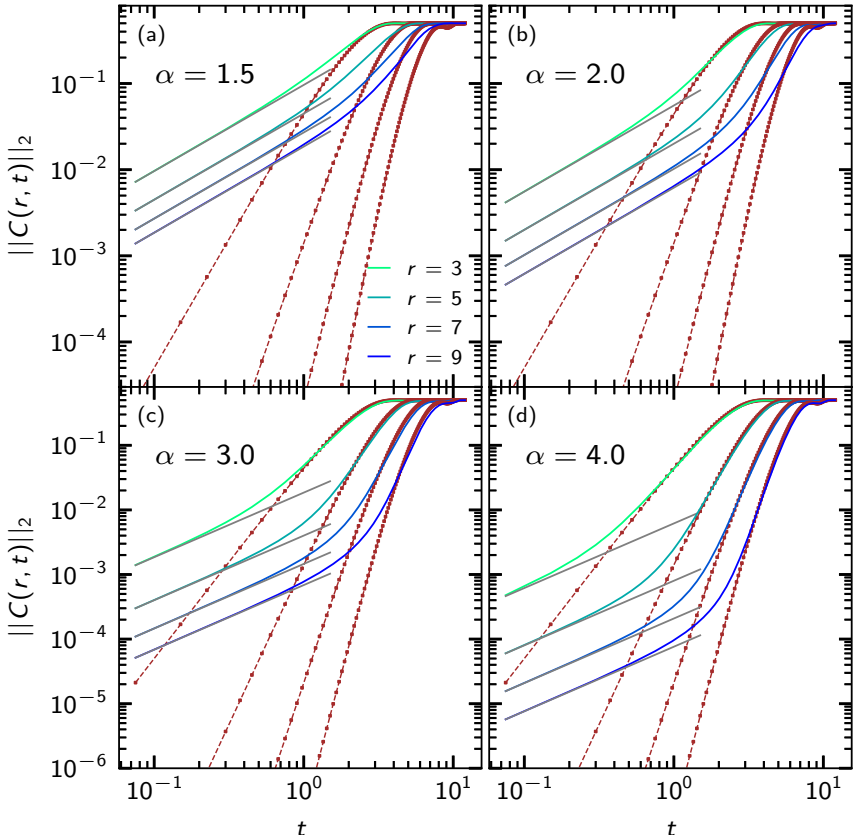

FIG. 3. Time evolution of $C_{2}(r, t)$ at fixed distance $r=3,5,7,9$ from $i=3$ and $\alpha=1.5,2.0,3.0,4.0$ for nearest-neighbor (dotted lines) and long-range interactions. Dashed lines are the leading order in perturbation theory given by Eq. (14). The system size is $L=14$.

For any finite $\alpha$, the operator norm given by Eq. (8) grows linearly in time and scales as $r^{-\alpha}$ at long distance and short times. We note that this perturbative behavior is true for any choice of the norm. In Fig. 3, the exact time evolution (colored lines) is compared to the leading order given by Eq. (14) (gray straight lines), yielding excellent agreement at short times.

On the other hand, Eq. (12) yields a very different behavior when interactions are limited to only nearest neighbors. In the short-range limit $\alpha \rightarrow \infty$, the support of the nested commutator $\left[\hat{H}, \hat{S}_{i}^{z}\right]_{m}$ grows by one lattice site at each $m$ term, which makes $\left[\left[\hat{H}, \hat{S}_{i}^{z}\right]_{m}, \hat{S}_{i+r}^{z}\right]$ vanish for $m<r$. This can be seen more clearly by looking at the first term in the expansion $\left[\hat{H}, \hat{S}_{i}^{z}\right]_{1}=i\left(\hat{S}_{i}^{x} \hat{S}_{i+1}^{y}-\hat{S}_{i}^{y} \hat{S}_{i+1}^{x}\right)+i\left(\hat{S}_{i-1}^{x} \hat{S}_{i}^{y}-\hat{S}_{i-1}^{y} \hat{S}_{i}^{x}\right)$, which has support only on sites $i-1, i, i+1$ and, therefore, $\left[\left[\hat{H}, \hat{S}_{i}^{z}\right]_{1}, \hat{S}_{i+r}^{z}\right]$ vanishes as long as $r>1$. Higher-order terms in the $\mathrm{BCH}$ formula for $r>1$ only become nonzero if a string of nontrivial Pauli matrices of length $r$ is generated between sites $i$ and $i+r$, and thus the leading order in the $\mathrm{BCH}$ formula for nearest-neighbor interactions reads

$$
\left[\hat{S}_{i}^{z}(t), \hat{S}_{i+r}^{z}\right] \propto \frac{t^{r}}{r !} \hat{\mathcal{O}}(1)
$$

where $\hat{\mathcal{O}}(1)$ is given by the operator norm of a sum of Pauli strings of length $r$. In other words, at short times and outside the light cone, the operator norm grows as a power law in time with an exponent given by the distance between the two operators. This is a quite general result, valid for any pair of local operators that are separated by a distance $r$ larger than the support of the most extended term in the Hamiltonian. In Fig. 6, the exact time evolution of Eq. (8) for the $X X X$ shortrange model is compared to the perturbation theory result

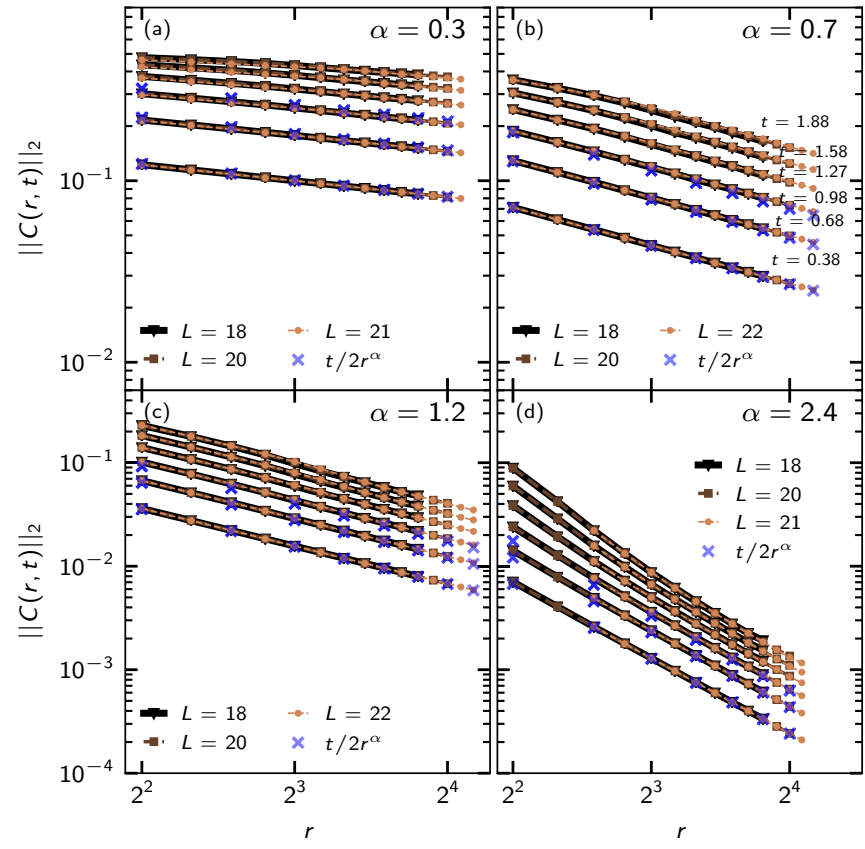

FIG. 4. Commutator as a function of distance $r$ from site $i=3$ at times $t=0.38,0.68,0.98,1.27,1.58,1.88$ (descending order in the plots). Crosses denote the asymptotic form given by Eq. (14). The overall behavior seems to be power law at any fixed time. The trends of the smaller sizes are followed off by the larger ones.

given by Eq. (15). The power-law growth $C_{2}(r, t) \propto t^{r}$ is in excellent agreement with the exact calculation at short times.

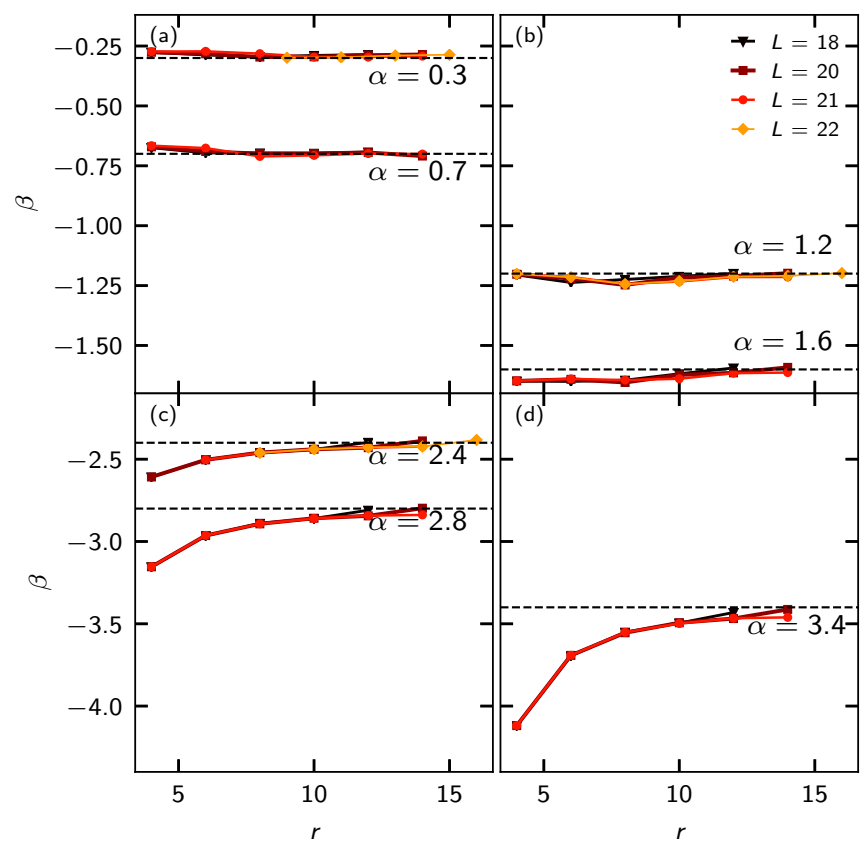

FIG. 5. Tail exponent of long-distance decay (see Fig. 4) computed from the discrete logarithmic derivative $\beta=\partial_{\ln r} \ln C_{2}(r, t)$ as a function of distance $r$ for different system sizes $L$ at fixed time $t=0.67$. The asymptotic exponent $\beta$ converges towards the interaction exponent $\alpha$ (dashed horizontal lines) for all values of $\alpha$ at long distances. 


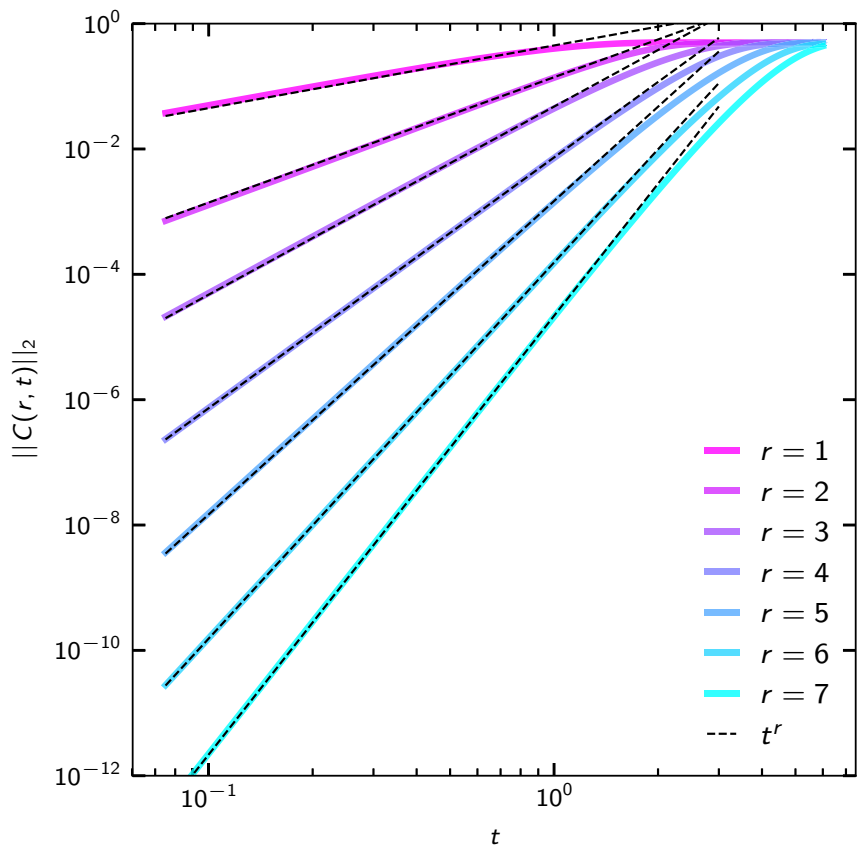

FIG. 6. Time evolution of the operator norm $C_{2}(r, t)$ at fixed distance $r$ for the nearest-neighbor $X X X$ chain with system size $L=18$. Black dashed lines are power-law fits $C_{2}(r, t) \sim t^{r}$ for the corresponding $r$. This is the behavior obtained in Eq. (15) from perturbation theory.

\section{Role of the nearest-neighbor part of the Hamiltonian at large $\alpha$}

For large values of $\alpha$, there is a significant speedup of the growth of the commutator norm and a clear departure from the linear growth of $C_{2}(r, t)$ at intermediate times (cf. Fig. 2, lower panels). On the other hand, when $\alpha$ is large enough, the commutator $C_{2}(r, t)$ is expected to exhibit a similar behavior as a short-range interacting system, which corresponds to the limit $\alpha \rightarrow \infty$ in Eq. (2). The Hamiltonian of the long-range model contains the nearest-neighbor part plus longer-distance couplings, decaying as $1 / r^{\alpha}$, which are strongly suppressed for $\alpha \gg 1$. Therefore, a dominant effect of the nearest-neighbor part is expected for large $\alpha[13,22]$. In Fig. 3, we compare $C_{2}(r, t)$ for the long-range model (full colored lines) to the nearest-neighbor model (red dashed lines) at fixed distances $r$. At short times, the long-range model shows the perturbative growth $r^{-\alpha} t / 2$ for all $\alpha$, and speeds up at intermediate times. The initial growth is significantly faster than in the case of nearest-neighbor interactions. For nearest-neighbor interactions, the operator norm $C_{2}(r, t)$ grows much faster due to the large power law $\propto t^{r}$. Therefore, at later times and for $\alpha>1$, the nearest-neighbor part catches up and dominates the overall growth of the commutator and leads to an asymptotic linear light cone.

Focusing only on large $\alpha$, the operator norm of the commutator exhibits two kinds of grows: linear at short times (see Fig. 2) and short range like at intermediate times (see Fig. 3). The short-range time evolution of $C_{2}(r, t)$ is well characterized by $(t / r)^{r}$ [31], while the long-range part is described by
Eq. (14), i.e., $t / r^{\alpha}$, in the limit $t \ll 1$. These two results can be combined into a single expression,

$$
C_{2}(r, t) \propto \frac{t}{2 r^{\alpha}}+\mathcal{O}(1)\left(\frac{t}{r}\right)^{r} .
$$

At short times, the linear term on the right-hand side is always dominant; at intermediate times, the second term become dominant and the dynamics is short-range like.

At long distances, there is a clear transition from a linear light cone for $\alpha>1$ to a power-law light cone at $\alpha<1$, which can be understood with the following reasoning. The asymptotic form of $C_{2}(r, t)$ in Eq. (16) grows monotonically and the two terms compete. The light cone is given by the set of times $t_{\theta}(r)$ as a function of distance $r$, for which $C_{2}(r, t)$ reaches a threshold value $\theta$, i.e., $C_{2}\left(r, t_{\theta}(r)\right)=\theta$. It is clear that $t_{\theta}(r) \leqslant t_{c}(r)=2 \theta r^{\alpha}$ since this is the time the first (linear in $t$ ) term needs to reach the threshold. If the second (power law in $t$ ) term reaches the threshold first, we get a linear light cone; otherwise, we get a power-law light cone. We can estimate the power-law term at long distances and $t \leqslant t_{c}$ by $t^{r} / r^{r} \leqslant t_{c}^{r} / r^{r}=\left(2 \theta r^{\alpha}\right)^{r} / r^{r}$. Therefore, at $t_{c}$, this term diverges for $\alpha>1$ and $r \rightarrow \infty$, and overwhelms the linear term, leading to a linear light cone $t_{\theta}(r) \propto r$. For $\alpha<1$ and $r \rightarrow \infty$, this term is irrelevant and we are left with a power-law light cone $t_{\theta}(r) \propto r^{\alpha}$. Analogously, the linear term is bounded by the time when the short-range front reaches the threshold, i.e., $t / 2 r^{\alpha} \leqslant r \theta^{1 / r} / 2 r^{\alpha}$. The bounding term vanishes in the limit $r \rightarrow \infty$ and $\alpha>1$, and diverges otherwise, which agrees with the bound to the short-range term. This behavior is consistent with the numerical observation in Fig. 1.

\section{E. Long-distance decay}

In Fig. 4, we analyze the behavior of $C_{2}(r, t)$ at long distances $r$ outside the light cone. It falls off as a power law at long distances, with an exponent that asymptotically approaches the interaction exponent $\alpha$ (see quantitative analysis using a discrete logarithmic derivative in Fig. 5). The same behavior was found previously [13] for the normalized Frobenius norm. This power-law decay $r^{-\alpha}$ is in perfect agreement with the prediction from perturbation theory in the short-time limit given by Eq. (14), and seems to be valid asymptotically outside the causal region.

This analysis confirms the validity of short-time perturbation theory (which is valid for any matrix norm) and shows that the asymptotic shape of the tails (outside the causal region) of long-range interacting spin chains is given by $C_{2}(r, t) \propto t / r^{\alpha}$.

\section{CONCLUSIONS}

The operator norm $C_{2}(r, t)$ of the commutator given by Eq. (1) has been examined in the $X X X$ chain with long-range interactions falling off as a power law $r^{-\alpha}$ with distance $r$ and interaction exponent $\alpha$. In order to reach large enough systems to check the convergence of our results with system size, we introduce a Krylov space method for the direct calculation of the operator norm of the commutator $C(r, t)$. We find a 




FIG. 7. Spreading of operator norm $C_{2}(x, t)=\left\|\left[S_{i}^{z}(t), S_{x}^{x}\right]\right\|_{2}$ in the $X Y Z$ model (B1) with $i=3$, over time and space for $\alpha=$ $0.4,0.8,1.3,2.5$. Continuous lines are contour lines given by the equation $\|C(x, t)\|=\theta$ with $\theta=0.1,0.2,0.25,0.3,0.35$ (marked as lines in the color bar in increasing order). System size is $L=14$. The shape of the "light cone" and their contour lines are very similar to the ones discussed in the main text (see Fig. 1).

linear growth in $t$ at early time $t$ and a long-distance decay outside the causal region given by $r^{-\alpha}$. Both the normalized Frobenius norm (directly related to OTOCs) and operator norm, which correspond to the average and fastest information spreading modes in the system, have the same asymptotic behavior (with different prefactors) of $t / r^{\alpha}$ at long distance and short time, which is strikingly different from systems with nearest-neighbor interactions, which instead exhibit a leading growth as a power law in time $\propto t^{r}$ at short times and long distances.

For $\alpha>1$, the information front is dominated by the contribution from the nearest-neighbor part of the Hamiltonian, which overtakes the initial linear growth of $C_{2}(r, t)$, inducing a linear light cone. These results are confirmed using a slightly different $X Y Z$ model in Appendix B.

Other theoretical studies predict a linear light-cone regime only for $\alpha>2$ [7,14,17-19,33], which is in conflict with our result $\alpha>1$. Since our numerics is limited to small sizes and short times, we cannot exclude a change in behavior at very long times and distances. Nevertheless, our exact calculations and the analytical asymptotic form from perturbation theory agree in a large parameter regime of our study (see, for example, Figs. 3 and 2). Since we also observe virtually no finite-size effects, our results provide no indication to expect a change of behavior in the asymptotic limit $t \rightarrow \infty, r \rightarrow \infty$.

We conclude that the findings from the study of out-of-time order correlators in Ref. [13] provide information about LiebRobinson bounds and agree with the behavior of the fastest spreading information mode in the system.

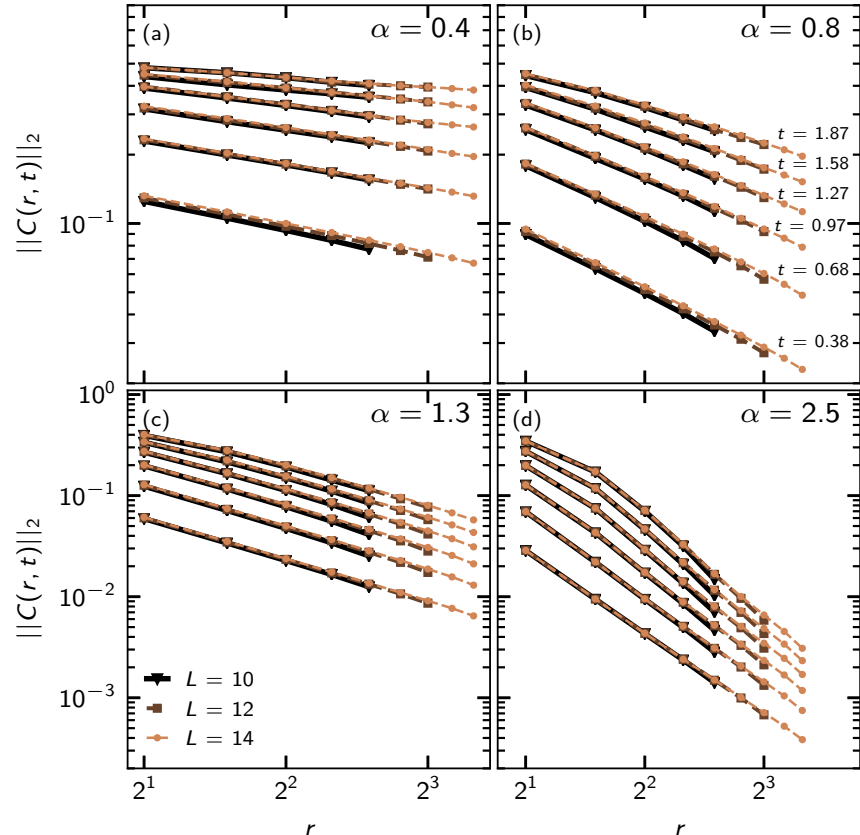

FIG. 8. Spatial decay of $C_{2}(r, t)$ in the $X Y Z$ model (B1) at fixed time $t=0.38,0.68,0.98,1.27,1.58,1.88$. Similarly to the $X X X$ model treated in the main text, there is a power-law decay (see Fig. 4).

\section{ACKNOWLEDGMENTS}

We acknowledge financial support from the Deutsche Forschungsgemeinschaft (DFG) through SFB 1143 (Project ID No. 247310070).

\section{APPENDIX A: PERTURBATIVE TREATMENT AT SHORT TIMES}

In Sec. III C, the Baker-Campbell-Hausdorff $(\mathrm{BCH})$ formula was employed for treating the time evolution of Eq. (1) at short times. From this analysis, the leading order of the operator norm $C_{2}(r, t) \approx t^{r} / r$ ! was obtained. In Fig. 6, the exact time evolution $C_{2}(r, t)$ is shown along with the leading order in the $\mathrm{BCH}$ formula (dashed lines), with excellent agreement at short times.

\section{APPENDIX B: RESULTS FOR $X Y Z$ MODEL}

In order to test the universality of the results presented in the main text, we have also performed similar calculations for the long-range $X Y Z$ Heisenberg model,

$$
H=\sum_{i<j} \frac{1}{|i-j|^{\alpha}}\left(J_{x} \hat{S}_{i}^{x} \hat{S}_{j}^{x}+J_{y} \hat{S}_{i}^{y} \hat{S}_{j}^{y}+J_{z} \hat{S}_{i}^{z} \hat{S}_{j}^{z}\right),
$$

with parameters $J_{x}=0.9, J_{y}=1.2, J_{z}=0.7$. The $X X X$ model study in the main text is recovered by setting $J_{x}=J_{y}=J_{z}=$ 1. The $X Y Z$ model does not have U(1) symmetry; therefore, the Hamiltonian is not block diagonal and we must deal with the full Hilbert space dimension $\mathcal{N}=2^{L}$. Analogously to the main text, we study the operator norm of the commutator,

$$
C_{2}(r, t)=\left\|\left[\hat{S}_{i}^{z}(t), \hat{S}_{i+r}^{x}\right]\right\|_{2} .
$$






FIG. 9. Time evolution of $C_{2}(r, t)$ in the $X Y Z$ model (B1) at fixed distance $r_{0}$ and system size $L=14$. Dot-dashed lines are the asymptotic form $t / 4 r^{\alpha}$ at short times $t<1$. The overall behavior is again similar to what was found for the $X X X$ model (see Fig. 2).

The difference compared to Eq. (8) lies in the static operator that is now $\hat{S}_{i+r}^{x}$. The time evolution and operator norm computation are carried out using exact diagonalization.

We analyze the behavior of Eq. (B2) as a function of both $r$ and $t$. In Fig. 7, the causal regions of $C_{2}(r, t)$ are shown. For all values of $\alpha$, the overall shape of the causal region is the same as for the $X X X$ long-range model (see Fig. 1).
Small $\alpha$ exhibit fast spreading with power-law causal regions and large $\alpha$ approach the linear light-cone limit $\alpha \rightarrow \infty$. The long-distance decay is also similar to what has been found in the main text (see Figs. 8 and 4), where outside the light "cone," there is a power-law decay of $C_{2}(r, t)$. Time evolution at fixed distance is displayed in Fig. 9 and is compatible with linear growth at short times $t<1$, which was also found in the $X X X$ version (see Fig. 2). In conclusion, the main features of both operator norms discussed in the main text are the same when considering a different commutator $\left[\hat{S}_{i}^{z}(t), \hat{S}_{i+r}^{x}\right]$ and a different long-range model, namely, the long-range $X Y Z$ model. As expected, only the interaction exponent $\alpha$ is crucial for characterizing the dynamics of the commutator given by Eq. (1).

Applying perturbation theory given by Eq. (12) up to first order, we get the following expression for the commutator when $r>0$ :

$$
\left[\hat{S}_{i}^{z}(t), \hat{S}_{i+r}^{x}\right]=\frac{t}{r^{\alpha}} S_{i+r}^{z} S_{i}^{x}+\mathcal{O}\left(t^{2}\right),
$$

yielding the following asymptotic form for the operator norm:

$$
\left\|\left[\hat{S}_{i}^{z}(t), \hat{S}_{i+r}^{x}\right]\right\|_{2}=\frac{t}{4 r^{\alpha}}+\mathcal{O}\left(t^{2}\right) .
$$

In Fig. 9, this asymptotic form is compared with the exact time evolution, yielding very good agreement. In conclusion, the $\mathrm{BCH}$ formula also predicts the short-time behavior for the $X Y Z$ model.
[1] A. Nahum, S. Vijay, and J. Haah, Operator Spreading in Random Unitary Circuits, Phys. Rev. X 8, 021014 (2018).

[2] V. Khemani, A. Vishwanath, and D. A. Huse, Operator Spreading and the Emergence of Dissipative Hydrodynamics under Unitary Evolution with Conservation Laws, Phys. Rev. X 8, 031057 (2018).

[3] C. W. von Keyserlingk, T. Rakovszky, F. Pollmann, and S. L. Sondhi, Operator Hydrodynamics, OTOCs, and Entanglement Growth in Systems without Conservation Laws, Phys. Rev. X 8, 021013 (2018).

[4] E. H. Lieb and D. W. Robinson, The finite group velocity of quantum spin systems, Commun. Math. Phys. 28, 251 (1972).

[5] M. B. Hastings, An area law for one-dimensional quantum systems, J. Stat. Mech. (2007) P08024.

[6] J. Eisert, M. Cramer, and M. B. Plenio, Colloquium: Area laws for the entanglement entropy, Rev. Mod. Phys. 82, 277 (2010).

[7] M. B. Hastings and T. Koma, Spectral gap and exponential decay of correlations, Commun. Math. Phys. 265, 781 (2006).

[8] B. Nachtergaele and R. Sims, Lieb-robinson bounds and the exponential clustering theorem, Commun. Math. Phys. 265, 119 (2006).

[9] S. Bravyi, M. B. Hastings, and F. Verstraete, Lieb-Robinson Bounds and the Generation of Correlations and Topological Quantum Order, Phys. Rev. Lett. 97, 050401 (2006).

[10] A. M. Kaufman, M. E. Tai, A. Lukin, M. Rispoli, R. Schittko, P. M. Preiss, and M. Greiner, Quantum thermalization through entanglement in an isolated many-body system, Science $\mathbf{3 5 3}$, 794 (2016).
[11] M. Kastner, N-scaling of timescales in long-range $N$-body quantum systems, J. Stat. Mech.: Theory Expt. (2017) 014003.

[12] D. J. Luitz and Y. Bar Lev, Information propagation in isolated quantum systems, Phys. Rev. B 96, 020406(R) (2017).

[13] D. J. Luitz and Y. Bar Lev, Emergent locality in systems with power-law interactions, Phys. Rev. A 99, 010105(R) (2019).

[14] M. Foss-Feig, Z.-X. Gong, C. W. Clark, and A. V. Gorshkov, Nearly Linear Light Cones in Long-Range Interacting Quantum Systems, Phys. Rev. Lett. 114, 157201 (2015).

[15] Z.-X. Gong, M. Foss-Feig, S. Michalakis, and A. V. Gorshkov, Persistence of Locality in Systems with Power-Law Interactions, Phys. Rev. Lett. 113, 030602 (2014).

[16] A. Y. Guo, M. C. Tran, A. M. Childs, A. V. Gorshkov, and Z.$\mathrm{X}$. Gong, Signaling and scrambling with strongly long-range interactions, Phys. Rev. A 102, 010401(R) (2020).

[17] D. V. Else, F. Machado, C. Nayak, and N. Y. Yao, Improved Lieb-Robinson bound for many-body Hamiltonians with power-law interactions, Phys. Rev. A 101, 022333 (2020).

[18] M. C. Tran, A. Y. Guo, Y. Su, J. R. Garrison, Z. Eldredge, M. Foss-Feig, A. M. Childs, and A. V. Gorshkov, Locality and Digital Quantum Simulation of Power-Law Interactions, Phys. Rev. X 9, 031006 (2019).

[19] P. Hauke and L. Tagliacozzo, Spread of Correlations in LongRange Interacting Quantum Systems, Phys. Rev. Lett. 111, 207202 (2013). 
[20] L. F. Santos, F. Borgonovi, and G. L. Celardo, Cooperative Shielding in Many-Body Systems with Long-Range Interaction, Phys. Rev. Lett. 116, 250402 (2016).

[21] C.-F. Chen and A. Lucas, Finite Speed of Quantum Scrambling with Long Range Interactions, Phys. Rev. Lett. 123, 250605 (2019).

[22] B. Kloss and Y. B. Lev, Spin transport in a long-rangeinteracting spin chain, Phys. Rev. A 99, 032114 (2019).

[23] X. Chen and T. Zhou, Quantum chaos dynamics in long-range power law interaction systems, Phys. Rev. B 100, 064305 (2019).

[24] H. Geng, Non-local entanglement and fast scrambling in desitter holography, arXiv:2005.00021.

[25] K. Kim, S. Korenblit, R. Islam, E. E. Edwards, M.-S. Chang, C. Noh, H. Carmichael, G.-D. Lin, L.-M. Duan, C. C. J. Wang, J. K. Freericks, and C. Monroe, Quantum simulation of the transverse Ising model with trapped ions, New J. Phys. 13, 105003 (2011).

[26] R. Blatt and C. F. Roos, Quantum simulations with trapped ions, Nat. Phys. 8, 277 (2012).

[27] M. Saffman, T. G. Walker, and K. Mølmer, Quantum information with Rydberg atoms, Rev. Mod. Phys. 82, 2313 (2010).

[28] I. Bloch, J. Dalibard, and S. Nascimbène, Quantum simulations with ultracold quantum gases, Nat. Phys. 8, 267 (2012).

[29] D. Otten, S. Rubbert, J. Ulrich, and F. Hassler, Universal powerlaw decay of electron-electron interactions due to nonlinear screening in a Josephson junction array, Phys. Rev. B 94, 115403 (2016).

[30] V. Khemani, D. A. Huse, and A. Nahum, Velocity-dependent Lyapunov exponents in many-body quantum, semiclassical, and classical chaos, Phys. Rev. B 98, 144304 (2018).

[31] Z. Wang and K. R. A. Hazzard, Tightening the Lieb-Robinson bound in locally-interacting systems, PRX Quantum 1, 010303 (2020).

[32] D.-M. Storch, Mauritz van den Worm, and M. Kastner, Interplay of soundcone and supersonic propagation in lattice models with power law interactions, New J. Phys. 17, 063021 (2015).

[33] T. Matsuta, T. Koma, and S. Nakamura, Improving the LiebRobinson Bound for Long-Range Interactions, Ann. Henri Poincaré 18, 519 (2017).

[34] L. Cevolani, G. Carleo, and L. Sanchez-Palencia, Spreading of correlations in exactly solvable quantum models with longrange interactions in arbitrary dimensions, New J. Phys. 18, 093002 (2016).
[35] T. Zhou, S. Xu, X. Chen, A. Guo, and B. Swingle, Operator Lévy Flight: Light Cones in Chaotic Long-Range Interacting Systems, Phys. Rev. Lett. 124, 180601 (2020).

[36] T. Kuwahara and K. Saito, Strictly Linear Light Cones in LongRange Interacting Systems of Arbitrary Dimensions, Phys. Rev. X 10, 031010 (2020).

[37] M. C. Tran, C.-F. Chen, A. Ehrenberg, A. Y. Guo, A. Deshpande, Y. Hong, Z.-X. Gong, A. V. Gorshkov, and A. Lucas, Hierarchy of Linear Light Cones with Long-Range Interactions, Phys. Rev. X 10, 031009 (2020).

[38] M. C. Tran, A. Ehrenberg, A. Y. Guo, P. Titum, D. A. Abanin, and A. V. Gorshkov, Locality and heating in periodically driven, power-law-interacting systems, Phys. Rev. A 100, 052103 (2019).

[39] A. I. Larkin and Yu. N. Ovchinnikov, Quasiclassical method in the theory of superconductivity, J. Expt. Theor. Phys. 28, 2262 (1969).

[40] J. Maldacena, S. H. Shenker, and D. Stanford, A bound on chaos, J. High Energy Phys. 08 (2016) 106.

[41] D. A. Roberts and B. Swingle, Lieb-Robinson Bound and the Butterfly Effect in Quantum Field Theories, Phys. Rev. Lett. 117, 091602 (2016).

[42] S. H. Shenker and D. Stanford, Black holes and the butterfly effect, J. High Energy Phys. 03 (2014) 067.

[43] F. D. M. Haldane, Exact Jastrow-Gutzwiller ResonatingValence-Bond Ground State of the Spin-1/2 Antiferromagnetic Heisenberg Chain with $1 / r^{2}$ Exchange, Phys. Rev. Lett. 60, 635 (1988).

[44] B. S. Shastry, Exact Solution of an $S=1 / 2$ Heisenberg Antiferromagnetic Chain with Long-Ranged Interactions, Phys. Rev. Lett. 60, 639 (1988).

[45] C. Bartsch and J. Gemmer, Dynamical Typicality of Quantum Expectation Values, Phys. Rev. Lett. 102, 110403 (2009).

[46] A. Haar, The concept of measure in the theory of continuous groups, Ann. Math. 34, 147 (1933).

[47] K. Hémery, F. Pollmann, and D. J. Luitz, Matrix product states approaches to operator spreading in ergodic quantum systems, Phys. Rev. B 100, 104303 (2019).

[48] A. Nauts and R. E. Wyatt, New Approach to Many-State Quantum Dynamics: The Recursive-Residue-Generation Method, Phys. Rev. Lett. 51, 2238 (1983).

[49] T. J. Park and J. C. Light, Unitary quantum time evolution by iterative Lanczos reduction, J. Chem. Phys. 85, 5870 (1986).

[50] D. J. Luitz and Y. Bar Lev, The ergodic side of the many-body localization transition, Ann. Phys. 529, 1600350 (2017). 